\title{
EFEITOS DA EXTRAÇÃO VERDE ASSISTIDA POR ULTRASSOM COMO PRÉ- TRATAMENTO NA OBTENÇÃO DO ÓLEO ESSENCIAL E HIDROLATO DE AROEIRA VERMELHA (SCHINUS TEREBINTHIFOLIUS RADDI)
}

\author{
EFFECTS OF ULTRASOUND-ASSISTED GREEN EXTRACTION AS A PRE- \\ TREATMENT TO OBTAIN THE ESSENTIAL OIL AND RED MASTIC HYDROLATE \\ (SCHINUS TEREBINTHIFOLIUS RADDI)
}

Fabricia Alessandra Garcia Mello de Oliveira ${ }^{1}$; Carla Cristina Bueno ${ }^{2}$; Fabíola Adriana Garcia Mello Dyna ${ }^{3}$; Michelle Karine dos Santos Lembi ${ }^{4}$; Thainá Aparecida Rafael Silva ${ }^{5}$; Creir da Silva $^{6}$; Cristiane Mengue Feniman Moritz ${ }^{7}$; Otávio Akira Sakai ${ }^{8}$.

1. Mestranda em Sustentabilidade, Instituto Federal do Paraná (IFPR), Umuarama, Paraná, email: fabricia1610mello@ gmail.com. ORCID: https://orcid.org/0000-0001-5703-6627.

2. Mestranda em Sustentabilidade, Universidade Estadual de Maringá (UEM), Umuarama, Paraná, email: carlatecnalimentos4@gmail.com. ORCID: https://orcid.org/0000-0002-07878372

3. Mestranda em Sustentabilidade, Universidade Estadual de Maringá (UEM), Umuarama, Paraná, email: fabiola.dyna@ gmail.com. ORCID: https://orcid.org/0000-0001-7729-3460

4. Mestranda em Sustentabilidade, Instituto Federal do Paraná (IFPR), Umuarama, Paraná, email: michelle.lembi23@gmail.com. ORCID: https://orcid.org/0000 -0002-5217-186X

5. Mestranda em Sustentabilidade, Instituto Federal do Paraná (IFPR), Umuarama, Paraná, email: thaina.rafael1197@ gmail.com. ORCID: https://orcid.org/0000 -0002-7957-6664

6. Mestre em Sustentabilidade, Instituto Federal do Paraná (IFPR), Umuarama, Paraná, email: creirdasilva@gmail.com. ORCID: https://orcid.org/0000 -0002-1053-7608

7. Docente do Programa de Pós Graduação Stricto Sensu em Sustentabilidade do Instituto Federal do Paraná (IFPR) e Universidade Estadual de Maringá (UEM), Umuarama, Paraná, email: Cristiane.feniman@ yahoo.com.br. ORCID: https://orcid.org/0000 -0002-9114-2156

8. Docente do Programa de Pós Graduação Stricto Sensu em Sustentabilidade do Instituto Federal do Paraná (IFPR) e Universidade Estadual de Maringá (UEM), Umuarama, Paraná, email: otavio.sakai@ifpr.edu.br. ORCID: https://orcid.org/0000 -0002-3502-5107

\section{RESUMO}

A aroeira vermelha, conhecida como pimenta rosa, natural de alguns estados do Brasil, é uma planta medicinal que apresenta atividades biológicas como anti-inflamatório, antipirético e analgésico. $\mathrm{O}$ objetivo do estudo foi verificar a influência do pré-tratamento por ultrassom no rendimento da extração do óleo essencial e hidrolato. A extração do óleo essencial foi realizada pelo método Clevenger com pré-tratamento de ultrassom com 30, 50 e $70 \%$ de potência. Observou-se que na potência de $70 \%$ obteve um maior rendimento. Em relação ao hidrolato, não se teve diferença significativa na extração sem e com o tratamento do ultrassom. Também foi realizada a caracterização por meio da espectroscopia do infravermelho por transformada de Fourier - reflectância total atenuada (FTIR-ATR), sendo uma técnica de enorme aplicabilidade para diversos tipos de amostras. É visto que o método da extração verde tem contribuído amplamente na redução de resíduos e na inovação de técnicas mais sustentáveis. 


\section{PALAVRAS-CHAVE}

Aroeira vermelha; Rendimento; Caracterização; Método da extração verde

\section{ABSTRACT}

The red pepper, known as pink pepper, natural in some states of Brazil, is a medicinal plant that has biological activities such as anti-inflammatory, antipyretic and analgesic. The extraction of essential oil was performed by hydrodistillation with a Clevenger device with pretreatment to optimize the process by means of ultrasound. The aim of the study was to verify how the ultrasound pretreatment influences the yield of essential oil and hydrolate extraction, in which it was observed that at $70 \%$ potency it obtained a higher yield. In relation to the hydrolate, there was no significant difference in the extraction without and with the ultrasound treatment. The characterization was also performed using Fourier transform infrared spectroscopy - attenuated total reflectance (FTIR-ATR), being a technique of enormous applicability for several types of samples. It is seen that the green extraction method has largely contributed to reducing waste and innovating more sustainable techniques.

\section{KEY WORDS}

Red pepper; Yield; Characterization; Green extraction method

\section{INTRODUÇÃO}

As plantas medicinais são o recurso natural mais abundante para o tratamento de infecções microbianas e são uma fonte importante na busca por novos fármacos com atividades terapêuticas. Os estudos etnofarmacológicos realizados no Brasil apresentam várias espécies com propriedades antimicrobianas, que têm sido usadas empiricamente pela população, principalmente espécies que pertencem à família Anacardiaceae.

A Schinus terebinthifolius Raddi, popularmente conhecida como aroeira vermelha, é considerada uma planta medicinal cujo uso é difundido em todo o Brasil. Praticamente todas as suas partes são comumente usadas para fins medicinais, incluindo os frutos, as sementes, as folhas e as cascas do caule (Santos; Silva \& Caxito, 2015). Trata-se de uma árvore de 5 a 7 metros, com caule cilíndrico, aéreo e lenhoso, apresentando ramificação simpodial. Sua casca é rugosa, com estrias longitudinais e cor marrom acinzentada. As folhas duram muitos anos e possui coloração esverdeada, forte aroma e disposicionais aos caules. O caule jovem dessa árvore tem contorno circular um pouco ondulado (Azevedo; Quirino \& Bruno, 2015).

A aroeira vermelha é originária de forma natural no Leste e Nordeste da Argentina, no Leste do Paraguai, no Uruguai e nos estados brasileiros de Sergipe, Paraíba, Alagoas, Pernambuco, Rio Grande do Norte, Bahia, Espírito Santo, Mato Grosso do Sul, Minas Gerais, Paraná, Rio de Janeiro, Santa Catarina e São Paulo (Neves et al., 2016). Schinus terebinthifolius 
é uma espécie vegetal nativa brasileira, amplamente encontrada na costa leste brasileira (bioma da Mata Atlântica) e desperta grande interesse na área científica devido ao seu potencial terapêutico (Silva-Neto, 2018). A maior parte da produção da aroeira vermelha é explorada por meio do extrativismo, principalmente nos Estados do Espírito Santo, Rio de Janeiro e em algumas ilhas do Rio São Francisco, na divisa entre Alagoas e Sergipe. Nessas áreas, a colheita é realizada pelas comunidades locais (Neves et al., 2016).

Também conhecida como pimenta rosa, a aroeira vermelha tem alto valor comercial no mercado interno e externo, como condimento gourmet. De sua polpa, pode-se extrair o óleo essencial que também apresenta potencial para o mercado de cosméticos, levando-se em consideração de que a extração desse óleo em escala industrial deve-se atingir as exigências de pureza no mercado internacional (Neves et al., 2016).

$\mathrm{O}$ fruto da aroeira vermelha possui aspecto pequeno e avermelhado e o óleo essencial extraído apresenta uma gama de bioatividades (Carvalho et al., 2016), composto por metabólitos secundários, como compostos fenólicos e terpenos, associados às suas atividades biológicas. As aplicações biológicas dessa planta são conhecidas há muitos anos e suas propriedades foram descritas desde a primeira edição da Farmacopéia Brasileira, publicada em 1926. Tem sido utilizada na medicina popular como anti-inflamatório, antipirético, analgésico e como agente depurativo (Carvalho et al., 2013).

Os metabólitos ativos da aroeira vermelha, como flavonóides, saponinas, terpenos e taninos possuem desempenho em suas propriedades terapêuticas. Todos esses compostos bioativos são encontrados nas folhas, nos frutos, óleo essencial e cascas do caule da aroeira vermelha (Maia et al., 2021). Os compostos fenólicos presentes na aroeira vermelha atuam como bioativos naturais, na proteção contra doenças crônicas devido à sua atividade antioxidante (Shahidi \& Ambigaipalan, 2015).

O óleo essencial de aroeira vermelha é rico em monoterpenos, sesquiterpenos e taninos. Esses compostos desempenham ação bactericida e bacteriostática, podendo ser eficazes em alguns casos de resistências bacterianas em área hospitalar (Silva-Neto, 2018). Além disso, outros ativos como saponinas (possuem propriedades tensoativas) e flavonóides (capacidade de interação com radicais livres para formar compostos estáveis) retardam o envelhecimento das células pelas ações anti-inflamatórias e cicatrizantes (Silva-Neto, 2018). Há também metabólitos secundários alcalóides, que proporcionam o sabor amargo na planta (Silva-Neto, 


\section{sunisul}

2018). Segundo Uliana (2016), o óleo essencial de aroeira vermelha possui funções antibacterianas para infecções do trato urinário e outras doenças humanas causadas por microrganismos.

Os diversos métodos de extração e solventes existentes podem interferir na quantificação de compostos químicos encontrados em uma matriz vegetal. Dessa forma, cada processo de extração é adequado a um determinado composto de interesse (Queiroz, 2001). E, deve-se levar em consideração a conservação dos compostos ativos íntegros, para evitar a sua degradação em processos que envolvem altas pressões e temperatura. Assim, torna-se importante a escolha mais adequada do método para promover a separação dos compostos de interesse (Amaral et al., 2021).

As substâncias aromáticas das plantas são extraídas de diferentes partes do vegetal, majoritariamente por hidrodestilação ou arraste a vapor. Assim são obtidos os óleos essenciais e hidrolatos. O óleo essencial é a porção lipofílica obtida no processo de destilação e o hidrolato é a porção aquosa (Bakkali, et al., 2007).

A extração assistida por ultrassom tem sua relevância por aumentar o rendimento na extração sólido-líquido (Amaral et al., 2021). O fluído supercrítico também tem se mostrado promissor nas extrações sem solventes tóxicos e na substituição de sínteses convencionais, em prol de processos de extração mais limpos e menor geração de resíduos (Pagliari et al., 2015).

Para a aroeira vermelha diferentes formas extrativas podem ser obtidas utilizando frutos, caule e folhas da planta, com extração por meio da maceração e assistida por ultrassom, decocção e turboextração (Araujo, et al., 2017). Por extração líquido-líquido, podem ser obtidos extratos etanólicos, butanóicos e óleos essenciais (Araujo, et al., 2017). Apesar das substâncias ativas serem encontradas em toda a planta, há maior concentração na casca, folhas e frutos (Silva-Neto, 2018).

Durante a destilação (principal método de extração de óleos essenciais), os vapores de água e óleo essencial entram em contato antes que se condensam e gotejam nos receptores como duas fases separadas (Edris, 2009; Aćimović, et al. 2020). Como resultado, parte dos componentes oxigenados dos óleos essenciais (que são altamente aromáticos) tornam-se solúveis em água devido à sua capacidade de produzir hidrogênio (Edris, 2009), resultando no hidrolato, também conhecido como hidrossol, água floral (flores) ou aromática. 
Considerando que os óleos essenciais são produtos obtidos de atividade extrativista, é importante buscar o maior rendimento possível por meio das tecnologias limpas de extração (Palermo et al., 2021) e a aplicação dos co-produtos gerados, como é o caso do hidrolato. Apesar do hidrolato ser rico em compostos hidrofílicos e polares, com possíveis atividades biológicas, muitas vezes é descartado após a separação do óleo essencial (Bakkali, et al., 2007).

Os hidrolatos podem apresentar propriedades antioxidante, analgésica, antifúngica e antibacteriana, com possibilidade de serem utilizados na indústria agroquímica, cosmética ou farmacêutica, estando em ascensão comercial e seu uso valoriza o conceito de sustentabilidade (Bastos et al., 2016).

Considerando a importância do óleo essencial de espécies nativas brasileiras obtido de forma extrativista sustentável, com maior eficiência de processo e aproveitamento de coprodutos, o objetivo deste estudo foi verificar a influência de pré-tratamento por ultrassom no rendimento, características físico-química e química do óleo essencial do fruto e, propriedades físicas do hidrolato de aroeira vermelha (Schinus terebinthifolius Raddi).

\section{MATERIAL E MÉTODOS}

\subsection{Material}

Os frutos de aroeira vermelha foram coletados no dia 03 de maio de 2021 de uma unidade vegetal localizada na cidade de Umuarama-PR ( $23^{\circ} 45^{\prime} 51^{\prime \prime}$ Sul, 53 19' 6" Oeste). Os frutos selecionados foram separados dos galhos e folhas e armazenados sob congelamento (-18 $\left.{ }^{\circ} \mathrm{C}\right)$ por 95 dias, até a extração do óleo essencial.

\subsection{Pré-tratamento por ultrassom}

Os frutos foram separados em quatro partes, sendo uma parte como tratamento controle (sem pré-tratamento) e as demais três partes foram submetidas ao pré-tratamento de ultrassom com 30, 50 e $70 \%$ de potência de $950 \mathrm{~W}$.

Para cada tratamento foram utilizados 40 gramas de frutos de aroeira vermelha previamente macerados em graal de porcelana e adicionados de $240 \mathrm{~mL}$ de água deionizada. Nos três grupos de pré-tratamento foi utilizado o equipamento de ultrassom UCD-950, BIOBASE, com os parâmetros de pulso ON por dois segundos, pulso OFF por três segundos e tempo total de 35 minutos. 


\subsection{Extração do óleo essencial por hidrodestilação via Clevenger}

Em balão volumétrico de $500 \mathrm{ml}$ foram colocados os tratamentos separadamente e procedeu-se à hidrodestilação em aparelho tipo Clevenger por 1,5 horas (Oliveira, 2014), com posterior separação do óleo essencial e hidrolatos, armazenados sob refrigeração em frascos âmbar.

\subsection{Leitura do índice pH}

A leitura do índice $\mathrm{pH}$ foi aferida através de medidor portátil (Akso AK103®), o eletrodo para medição do $\mathrm{pH}$ foi inserido nas amostras de hidrolato em temperatura ambiente de $25^{\circ} \mathrm{C}$.

\subsection{Rendimento e densidade absoluta do óleo essencial}

$\mathrm{O}$ rendimento de óleo essencial foi determinado pesando-se a massa das amostras obtidas em balança semi-micro analítica (marca Shimadzu - modelo AUW220D) e cálculo da porcentagem em relação à massa inicial dos frutos.

A densidade absoluta do óleo essencial dos tratamentos foi realizada em triplicata por capilaridade (capilar de vidro 5 $\mu \mathrm{L}$ - Marca intraEND BLAUBRAND®), utilizando a razão da massa (g) pelo seu volume (mL) a $25^{\circ} \mathrm{C}$, de acordo com a Farmacopéia Brasileira (1988).

\subsection{Espectroscopia do infravermelho por transformada de Fourier - reflectância total atenuada (FTIR-ATR) para o óleo essencial de aroeira vermelha}

Os espectros FTIR-ATR das amostras de óleo essencial foram realizadas no espectrômetro de infravermelho (modelo Cary 630 FTIR, marca Agilent Technologies). O intervalo espectral foi de 4000 a $400 \mathrm{~cm}^{-1}$, varredura de 64 scans e $4 \mathrm{~cm}^{-1}$ de precisão. As análises foram realizadas em triplicata.

\subsection{Caracterização do hidrolato de aroeira vermelha e suas partículas dispersas}

Foi utilizado o analisador de partículas Litesizer TM 500 (Anton Paar Instrumentos, Grantz, Áustria), conforme os parâmetros estabelecidos por Liew et al. (2020).

Foi determinado o tamanho de partícula por medição da dispersão da luz dinâmica (Dynamic Light Scattering - DLS), com 200 corridas a 25, 30, 35 e $40^{\circ} \mathrm{C}$ em ângulo de $90^{\circ}$ e a $25^{\circ} \mathrm{C}$ em ângulo automático. Também foi medido o índice de polidispersão e o potencial zeta 
por dispersão de luz eletroforética (Eletrophoretic Light Scatterin - ELS) em $25{ }^{\circ} \mathrm{C}$ e 200 corridas.

\subsection{Análise estatística}

Os dados obtidos dos diferentes tratamentos de potência e temperaturas aplicados em relação ao raio hidrodinâmico, índice de polidispersão das partículas, temperatura e potencial zeta foram avaliados de acordo com o teste Tukey ao nível de 5\% de probabilidade de erro

\section{RESULTADOS E DISCUSSÃO}

\subsection{Rendimento e densidade absoluta do óleo essencial}

Dos resultados obtidos de rendimento e densidade do óleo essencial por meio da hidrodestilação assistida por ultrassom, foi possível observar a diferença de rendimento de óleo essencial quanto maior foi a potencial utilizado no pré-tratamento de ultrassom, conforme apresentados na tabela 1. Considerou-se que o ultrassom facilitou a saída dos compostos voláteis do material vegetal.

Tabela 1 - Rendimento e densidade do óleo essencial de aroeira vermelha, obtido por hidrodestilação assistida por ultrassom.

\begin{tabular}{|c|c|c|c|}
\hline $\begin{array}{c}\text { Pré-tratamento } \\
\text { com ultrassom } \\
\text { (potência de } 950 \mathrm{~W} \text { ) }\end{array}$ & $\begin{array}{c}\text { Volume }(\mathrm{mL}) \text { de } \\
\text { óleo essencial } \\
\text { obtido }\end{array}$ & Rendimento (\%) & Densidade $\left(\mathrm{g} \mathrm{mL}^{-1}\right)$ \\
\hline Controle & 0,65 & 1,58 & $0,9360 \pm 0,0480$ \\
\hline $30 \%$ & 0,60 & 1,49 & $1,0013 \pm 0,0880$ \\
\hline $50 \%$ & 0,83 & 2,05 & $0,9093 \pm 0,0684$ \\
\hline $70 \%$ & 0,90 & 2,50 & $0,9526 \pm 0,1380$ \\
\hline
\end{tabular}

Fonte: Autores, 2021

Apesar do tratamento de potência $30 \%$ ter apresentado menor rendimento de óleo essencial comparado com o controle, os outros dois tratamentos apresentaram maiores rendimentos, com aumento de 29,75 e 58,23 \% para os tratamentos de 50 e $70 \%$ de potência, respectivamente. No entanto, houve uma diferença significativa na densidade absoluta dos 


\section{¿unisul}

óleos essenciais, provavelmente em consequência da extração de compostos de maior peso molecular que não foram extraídos no tratamento controle.

\subsection{Espectros FTIR do óleo essencial}

A espectroscopia de infravermelho tem grande aplicabilidade nos dias contemporâneos por serem confiáveis, rápida e de fácil manipulação, possui dois feixes de luz, sendo possíveis para amostras em fases líquidas, sólidas ou gasosas. A reflectância total atenuada (ATR) é um procedimento para atingir os espectros FTIR de determinados tipos de amostras, com a radiação do meio mais denso para o menos denso e com a sua finalidade qualitativa para grupos funcionais de moléculas (Simões, 2018) e pode fornecer informações importantes sobre as interações de ligações intra e intermolecular. Os óleos essenciais dos frutos da aroeira vermelha extraídos por diferentes condições de pré-tratamento com ultrassom (controle, 30\%, 50\% e $70 \%$ ) foram caracterizados por FTIR e os espectros estão apresentados nas Figuras 1 e 2.

Figura 1 - Espectro no infravermelho do óleo essencial de aroeira vermelha extraído por hidrodestilação e com diferentes potências de ultrassom como pré-tratamento com intervalo espectral de 3200 a $2600 \mathrm{~cm}^{-1}$.

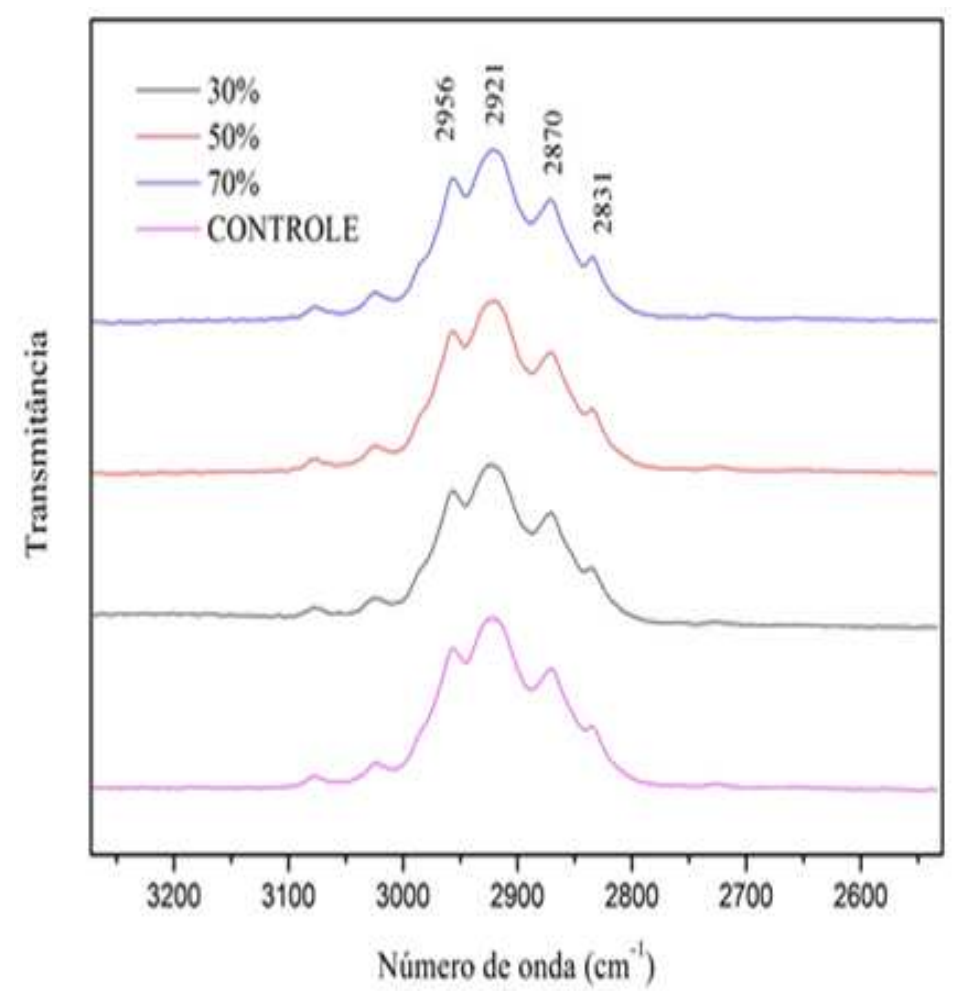

Fonte: Autores, 2021 


\section{¿unisul sim}

Figura 2 - Espectro no infravermelho do óleo essencial de aroeira vermelha extraído por hidrodestilação e com diferentes potências de ultrassom como pré-tratamento, com intervalo espectral de 1700 a $500 \mathrm{~cm}^{-1}$.

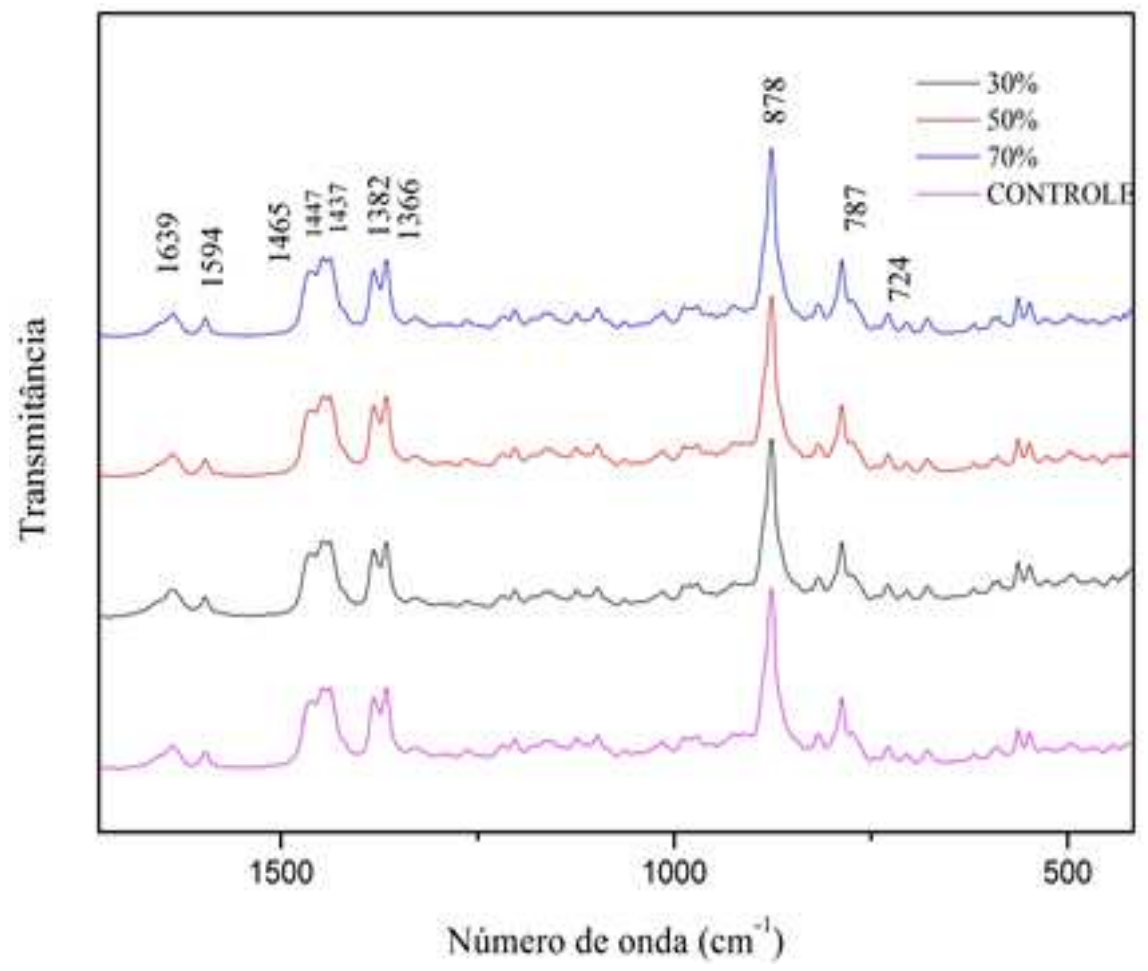

Fonte: Autores, 2021

Nos espectros de infravermelho mostrados nas Figuras 1 e 2, observou-se que as principais bandas obtidas são similares (intensidade e posição) tanto para o controle (somente hidrodestilação) quanto as diferentes potências utilizadas no pré-tratamento de ultrassom. Ou seja, pode-se inferir que não houve diferença de alteração dos compostos químicos presentes no óleo essencial da aroeira quando se utiliza o ultrasonicador em diferentes potências.

$\mathrm{Na}$ Figura 1, o pico em $2956 \mathrm{~cm}^{-1}$ está associado ao estiramento simétrico $\mathrm{CH}_{3}$. O pico em $2921 \mathrm{~cm}^{-1}$ corresponde a banda dos alcenos $(\mathrm{C}-\mathrm{H})$. As outras duas bandas de transmissão em $2870 \mathrm{~cm}^{-1}$ e $2831 \mathrm{~cm}^{-1}$ estão relacionadas aos estiramentos simétrico metil C-H e metóxi C$\mathrm{CH}_{3}$, respectivamente (Nandiyant; Oktiani \& Ragadhita, 2019). A banda tripleto $\left(2956 \mathrm{~cm}^{-1}\right.$, $2921 \mathrm{~cm}^{-1}$ e $2870 \mathrm{~cm}^{-1}$ ) pode representar alcenos aromáticos e os compostos monoterpenos (Silva, 2015), que são majoritários no óleo essencial de aroeira vermelha. 
Na Figura 2, a banda observada em $1465 \mathrm{~cm}^{-1}$ corresponde as vibrações de flexão $\mathrm{CH}_{2}$ e $\mathrm{CH}_{3}$ do grupo alifático. Outras bandas aparecem em $1382 \mathrm{~cm}^{-1} \mathrm{e} 724 \mathrm{~cm}^{-1}$ associadas à flexão das vibrações $\mathrm{CH}_{2}$ e $\mathrm{CH}_{3}$. As bandas de transmitância em $1639 \mathrm{~cm}^{-1}, 1437 \mathrm{~cm}^{-1}$ e $1382 \mathrm{~cm}^{-1}$ correspondem ao estiramento $\mathrm{C}=\mathrm{C}$ das vibrações dos alcanos, $\mathrm{C}-\mathrm{H}$ vibrações de flexão dos alcanos e O-H vibrações dos fenóis, respectivamente (Berechet et al., 2015; Benoudjit et al., 2020). A região de 1500 a $600 \mathrm{~cm}^{-1}$ é conhecida como região de “impressão digital” do espectro, que inclui várias bandas acopladas. Nessa região encontram-se as bandas $878 \mathrm{~cm}^{-1}$, $787 \mathrm{~cm}^{-1}$ e $724 \mathrm{~cm}^{-1}$, que estão associadas à vibração fora do plano C-H do anel aromático (Nandiyant; Oktiani; Ragadhita, 2019). Tais bandas podem representar compostos majoritários como $\delta$-3-careno, limoneno, $\alpha$-felandreno, $\alpha$-pineno, mirceno e sesquiterpenos relatados no estudo de Picolloto et al. (2020).

\subsection{Caracterização física do hidrolato}

O hidrolato é considerado uma parte do óleo essencial dissolvido em água proveniente do processo da destilação. Tem pH ácido entre pH 3,5 e 6,5 e pode ser considerado um colóide, uma vez que as partículas de óleo essencial em suspensão estão carregadas em água. O tamanho hidrodinâmico de dispersão pode variar entre $1 \mathrm{~nm}<\mathrm{d}<10 \mathrm{~nm}$ dependendo do tipo de solução, temperatura, concentração, ângulo de análise.

Neste estudo foi possível avaliar a influência da potência do ultrassom no raio hidrodinâmico das partículas do hidrolato (Tabela 2), o índice de polidispersão (Tabela 3) e o potencial zeta (Tabela 4$)$.

Tabela 2 - Tamanho de partícula $(\mathrm{nm})$ do hidrolato de aroeira vermelha extraído por hidrodestilação e com diferentes potências de ultrassom como pré-tratamento.

\begin{tabular}{|c|c|c|c|c|c|}
\hline \multirow{2}{*}{ Tratamentos } & \multirow{2}{*}{$\begin{array}{c}\text { Ângulo } \\
\text { Automático } \\
2^{\circ} \mathrm{C}\end{array}$} & \multicolumn{4}{|c|}{ Ângulo de $90^{\circ}$} \\
\hline & & $25^{\circ} \mathrm{C}$ & $30{ }^{\circ} \mathrm{C}$ & $35^{\circ} \mathrm{C}$ & $40{ }^{\circ} \mathrm{C}$ \\
\hline Controle & $\begin{array}{c}1.294,9 \mathrm{aA} \pm \\
606,6\end{array}$ & $\begin{array}{c}754,4 \mathrm{aA} \pm \\
439,1\end{array}$ & $\begin{array}{c}570,8 \mathrm{aA} \pm \\
1.778,4\end{array}$ & $\begin{array}{c}1.800,1 \mathrm{aA} \pm \\
1.629,9\end{array}$ & $\begin{array}{c}2.913,3 \mathrm{aA} \pm \\
1.480,0\end{array}$ \\
\hline
\end{tabular}




\section{GESTÃO \& SUSTENTABILIDADE}

AMBIENTAL

\section{unisul}

\begin{tabular}{cccccc} 
Potência de & $647,1 \mathrm{aA} \pm$ & $389,9 \mathrm{aA} \pm$ & $858,6 \mathrm{aA} \pm$ & $1.206,5 \mathrm{aA} \pm$ & $617,8 \mathrm{aA} \pm$ \\
$30 \%$ & 341,9 & 1,92 & $1.672,1$ & $3.432,6$ & 202,3 \\
& & & & & \\
Potência de & $582,6 \mathrm{aA} \pm$ & $362,5 \mathrm{aA} \pm$ & $610,2 \mathrm{aA} \pm$ & $29.641,9 \mathrm{aA}$ & $4.337,6 \mathrm{aA} \pm$ \\
$50 \%$ & $7.785,6$ & 43,5 & $1.599,2$ & $\pm 32.391,1$ & $7.122,6$ \\
& & & & & \\
Potência de & $736,1 \mathrm{aA} \pm$ & $767,4 \mathrm{aA} \pm$ & $595,5 \mathrm{aA} \pm$ & $486,8 \mathrm{aA} \pm$ & $445,9 \mathrm{aA} \pm$ \\
$70 \%$ & $6.176,7$ & 190,2 & 54,9 & 64,2 & 190,1 \\
\hline
\end{tabular}

Letras minúsculas iguais na mesma linha indicam igualdade significativa a $95 \%$. Letras maiúsculas iguais na mesma coluna indicam igualdade significativa a $95 \%$. Fonte: Autores, 2021

Foi analisado neste estudo, se a potência do ultrasonicador contribuiu para a alteração do raio hidrodinâmico das partículas dispersas no hidrolato. No entanto, pela análise estatística dos dados do espalhamento dinâmico de luz (DLS) não foi constatada diferença significativa a $5 \%$, sem influência dos fatores potência de ultrassom e temperatura, apesar de constatadas diferentes variâncias para as repetições.

Tabela 3 - Índice de polidispersão (\%) das partículas do hidrolato de aroeira vermelha extraído por hidrodestilação e com diferentes potências de ultrassom como pré-tratamento.

\begin{tabular}{|c|c|c|c|c|c|}
\hline \multirow{2}{*}{ Tratamentos } & \multirow{2}{*}{$\begin{array}{c}\text { Ângulo } \\
\text { Automático } \\
\mathbf{2 5}^{\circ} \mathrm{C}\end{array}$} & \multicolumn{4}{|c|}{ Ângulo de $90^{\circ}$} \\
\hline & & $25^{\circ} \mathrm{C}$ & $30^{\circ} \mathrm{C}$ & $35^{\circ} \mathrm{C}$ & $40^{\circ} \mathrm{C}$ \\
\hline Controle & $\begin{array}{c}42,18 \pm \\
11,15\end{array}$ & $\begin{array}{c}27,61 \mathrm{aA} \pm \\
1,20\end{array}$ & $\begin{array}{c}45,31 \mathrm{aA} \pm \\
41,56\end{array}$ & $\begin{array}{c}44,65 \mathrm{aAB} \pm \\
3,94\end{array}$ & $\begin{array}{c}38,10 \mathrm{aA} \pm \\
5,50\end{array}$ \\
\hline $\begin{array}{l}\text { Potência de } \\
30 \%\end{array}$ & $26,42 \pm 0,76$ & $\begin{array}{c}26,95 \mathrm{aA} \pm \\
2,13\end{array}$ & $\begin{array}{c}30,39 \mathrm{aA} \pm \\
5,58\end{array}$ & $\begin{array}{c}32,85 \mathrm{aA} \pm \\
5,28\end{array}$ & $\begin{array}{c}39,97 \mathrm{aA} \pm \\
41,39\end{array}$ \\
\hline $\begin{array}{l}\text { Potência de } \\
50 \%\end{array}$ & $\begin{array}{c}49,75 \pm \\
30,24\end{array}$ & $\begin{array}{c}24,88 \mathrm{aA} \pm \\
1,14\end{array}$ & $\begin{array}{c}34,94 \mathrm{aA} \pm \\
3,72\end{array}$ & $\begin{array}{c}63,45 \mathrm{bB} \pm \\
7,20\end{array}$ & $\begin{array}{c}47,42 \mathrm{abA} \pm \\
6,65\end{array}$ \\
\hline $\begin{array}{l}\text { Potência de } \\
70 \%\end{array}$ & $37,05 \pm 7,73$ & $\begin{array}{c}25,74 \mathrm{aA} \pm \\
5,99\end{array}$ & $\begin{array}{c}41,83 \mathrm{aA} \pm \\
25,05\end{array}$ & $\begin{array}{c}38,67 \mathrm{aA} \pm \\
12,17\end{array}$ & $\begin{array}{c}35,38 \mathrm{aA} \pm \\
3,82\end{array}$ \\
\hline
\end{tabular}

Letras minúsculas iguais na mesma linha indicam igualdade significativa a $95 \%$.

Letras maiúsculas iguais na mesma coluna indicam igualdade significativa a $95 \%$.

Fonte: Autores, 2021 


\section{¿unisul}

Quanto ao índice de polidispersão das partículas no hidrolato houve variações estatisticamente significativas para o hidrolato aquecido a $35^{\circ} \mathrm{C}$, sendo maior índice de polidispersão para o pré-tratamento com ultrassom de $50 \%$.

$\mathrm{O}$ hidrolato de aroeira vermelha extraído sem tratamento do ultrassom apresentou $\mathrm{pH}$ 4,0 e para os pré-tratamentos de ultrassom com 30, 50 e 70\%, o hidrolato foi obtido com $\mathrm{pH}$ 4,2, 4,3 e 4,2, respectivamente. Desse modo, não houve uma diferença discrepante no pH dos hidrolatos provenientes de destilação com ultrassom assistida, o que pode ter contribuída na estabilidade do potencial Zeta $(\zeta)$, que foi negativo e próximo a zero para todos os tratamentos (Tabela 4), com igualdade significativa a 95\%, provavelmente pela presença de pólos negativos nas partículas (Fayada et al., 2015).

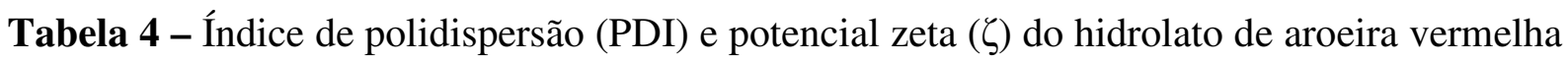
extraído por hidrodestilação e com diferentes potências de ultrassom como pré-tratamento.

\begin{tabular}{ccc}
\hline Tratamentos & Potencial zeta (Volts) & Desvio Padrão \\
\hline Controle & $-0,020154395 \mathrm{a}$ & 0,00028 \\
Potência de $30 \%$ & $-0,03025083 \mathrm{a}$ & 0,00028 \\
Potência de $50 \%$ & $-0,029162043 \mathrm{a}$ & 0,00144 \\
Potência de $70 \%$ & $-0,017763042 \mathrm{a}$ & 0,00772 \\
\hline
\end{tabular}

Letras minúsculas iguais na mesma coluna indicam igualdade significativa a $95 \%$.

Fonte: Autores, 2021

No amplo campo do desenvolvimento sustentável no setor industrial e outros, a implantação da química verde tem ganhado espaço com suas prioridades em redução da produção de resíduos, na diminuição de custos futuros, na eficiência de energia e com o surgimento de inovações tecnológicas entre os materiais usados e com a otimização nas metodologias dos seus processos (Barbosa, Rocha \& Teixeira, 2015). 


\section{CONCLUSÃO}

O fruto da aroeira vermelha, também conhecida como pimenta rosa, foi submetida ao processo de extração verde - hidrodestilação com pré-processamento por ultrassom $(30,50$ e $70 \%$ de potência de $950 \mathrm{~W}$ ) - para obtenção do óleo essencial. O tratamento de potência 30\% apresentou menor rendimento de óleo essencial comparado com o controle e os outros dois tratamentos $(50 \%$ e $70 \%)$ apresentaram maiores rendimentos $29,75 \%$ e $58,23 \%$, respectivamente. Por meio dos espectros de FTIR do óleo essencial pôde-se ser observado que não houve possivelmente alteração dos alcenos aromáticos, compostos monoterpenos $\delta$-3careno, limoneno, $\alpha$-felandreno, $\alpha$-pineno, mirceno e sesquiterpeno quando utilizado o ultrassom em diferentes potências. Em relação aos hidrolatos, o pH não apresentou variação, o que contribuiu para a estabilidade do potencial zeta, negativo e próximo a zero, com igualdade significativa a 95\%. Verificou-se que o raio hidrodinâmico dos hidrolatos não se alteraram em decorrência da potência e temperatura.

Conclui-se que a extração assistida por ultrassom associado a hidrodestilação, sendo uma forma de extração verde, foi eficaz para aumentar o rendimento do óleo essencial, e aproveitamento dos co-produtos contribuindo com o desenvolvimento de estratégias sustentáveis, o que abre oportunidade para que outros estudos sejam investigados.

\section{AGRADECIMENTOS}

Os autores agradecem o Laboratório de Análises Cromatográficas e Espectroscópicas IFPR, Campus Umuarama e Anton Paar Brasil.

\section{REFERÊNCIAS}

Aćimović, M. G.; Tešević, V. V.; Smiljanić, K.T.; Cvetković, M. T.; Stanković, J. M.; Kiprovski, B. M.; Sikora, V. S., (2020). Hydrolates - by-products of essential oil distillation:chemical composition, biological activity and potential uses. Advanced Technologies. v. 9, n. 2, p. 54-70. DOI: 10.5937/savteh2002054A

Amaral, R. A. G.; Tonhela, M. A.; Antonelli, R.; Okura, M. H.; Malpass, G. R. P.; Granato, A. C., (2021). Experimental design for ultrasound-assisted extraction of Schinus terebinthifolius. Research Society and Development, v. 10, n. 3. DOI: 10.33448/rsdv10i3.12872 
Araujo, J. C.; Mattos, L. L.; Paulo, L.M.; Silva, C.C. R.; Ferreira, R. P.; Sales, M. D.C., (2017). Análise de Métodos em Testes de Extratos de Própolis e Aroeira. In III Seminário Científico da FACIG, II Jornada de Iniciação Científica da FACIG, p.1-8.

Azevedo, C. F.; Quirino, Z. G. M.; Bruno, R. L. A., (2015). Estudo farmacobotânico de partes aéreas vegetativas de aroeira-vermelha Shinus terebinthifolius Raddi, Anacardiaceae). Revista Brasileira de Plantas Medicinais. v. 17, n. 1, p. 26-35. DOI: 10.1590/1983-084X/11_090

Asep Bayu Dani Nandiyanto, Rosi Oktiani and Risti Ragadhita, How to read and interpret FTIR spectroscope of organic materia, Indones. J. Sci. Technol. 4 (2019) 97-118.

Bakkali, F.; Averbeck, S.; Averbeck, D.; Idaomar, M., (2008). Biological effects of essential oils-a review. Food and Chemical Toxicology, v. 46, p. 446-475.

https://www.sciencedirect.com/science/article/abs/pii/S0278691507004541

Barbosa, L. S.; Rocha, G. P.; Teixeira, J. R. F., (2015). The principles of green chemistry and the analytical laboratories sustainability. Periódico Tchê Química, v. 12, n. 24.

Bastos, L. R.; Bernardes, P.C.; Carvalho, J.A.M.; Marques, C.S.; Pinheiro, P.F., (2016). Composição Química e Avaliação da Atividade Antimicrobiana do Óleo de Pimenta Rosa (Schinus terebinthifolius). In: V Semana de Engenharia Química UFES. Alegre, Espírito Santo. http://pdf.blucher.com.br.s3-sa-east1.amazonaws.com/chemicalengineeringproceedings/vsequfes2016/014.pdf

Berechet, M.D.; Calinescu, I.; Stelescu, M.D.; Manaila, E.; Craciun, G.; Purcareanu, B.; Mihaiescu, D.E.; Rosca, S.; Fudulu, A.; Niculescu-Aron, I.G.; et al. Composition of the essential oil of Rosa damascena Mill. cultivated in Romania. Rev. Chim. (2015), 66, 19861991

Carvalho, M.G.; Melo, A.G.N.; Aragão, C.F.S.; Raffin, F.N.; Moura, T.F.A.L., (2013).

Schinus terebinthifolius Raddi: chemical composition, biological properties and toxicity. Rev. Bras. Pl. Med., Botucatu, v. 15, n. 1, p.158-169. DOI: 10.1590/S1516-05722013000100022

Costa C., (2011). Avaliação da atividade antioxidante e antimicrobiana de extratos de Myracrodruon Urundeuva Allemão e Schinus terebinthifolius Raddi. Dissertação (Pós-

Graduação em Processos Interativos dos Órgãos e Sistemas) - Instituto de Ciências da Saúde. Universidade Federal da Bahia, Salvador, p. 66.

https://repositorio.ufba.br/ri/bitstream/ri/15632/1/Cinara\%20Costa.pdf

Edris, A. E., (2009). Identification and absolute quantification of the major water-soluble aroma components isolated from the hydrosols of some aromatic plants. Jeobp, v. 12, n. 2, p. 155 - 161. DOI: 10.1080/0972060X.2009.10643705

Farmacopéia Brasileira. 4. ed. São Paulo: Atheneu, 1988.

Fayad, S. J.; Ramos, B. G. Z.; Soldi, V.; Minatti, E.; (2015). Nanopartículas de proteína isolada de soja em água: Efeito da força iônica e das concentrações de proteína e surfactante. Quimica Nova, 38, 1, 91-96. DOI: 10.5935/0100-4042.20140295

Liew, S. N.; Utra, U.; Alias, A. K.; Tan, T. B.; Tan, C. P.; (2020). Yussof, N. S. Physical, morphological and antibacterial properties of lime essential oil nanoemulsions prepared via spontaneous emulsification method. Food Science and Technology, v. 128, p. 1-8. 
Maia, M. C.R.; Laurentino, C. S.; Carneiro, G.A.; Muniz, I. C. S.; Muniz, I. I. S.; Silva, I. A.; Reis, J. A.; Sultanun, R. F. S.; Vasconcelos, T. R. L. C.; Cordeiro, R. P., (2021). Propriedades terapêuticas da espécie Shinus terebinthifolius Raddi (aroeira-vermelha). Revista Eletrônica Acervo Saúde. v. 13, n. 4, p. 1-8. DOI: 10.25248/reas.e6791.2021

Neves, E. J. M.; Santos, A. M.; Gomes, J. B. V.; Ruas, F. G.; (2016). Ventura, J. A. Cultivo da aroeira-vermelha (Schinus terebinthifolius Raddi) para a produção de pimenta-rosa. Embrapa-Documentos (INFOTECA-E), v. 1, n. 1, p. 114-117.

Oliveira, L.F.M.; Oliveira JR, L.F.G.; Santos, M.C.; Narain, N.; Leite neta, M.T.S., (2014) Tempo de destilação e perfil volátil do óleo essencial de aroeira da praia (Schinus terebinthifolius) em Sergipe. Revista Brasileira de Plantas Medicinais, Campinas, v. 16, n. 2, p. 243-249.

Pagliari, A. B.; Bianchi, A. R.; Zanatta, J. S.; Orlando, T.; Emmerich, D. J.; Cunico, W., (2015). Synthesis of thiazolidinones and oxathiolones by supercritical fluid. Perspectiva, v. 39, n. 147, p. 43-50. https://www.uricer.edu.br/site/pdfs/perspectiva/147_524.pdf

Palermo, G. P. M.; Blanco, J. C.; Fernandes, M. C. A.; Sampaio, D. A.; castor neto, T. C.; Almeida, J. P. S.; Rodrigues, A. N. C., (2021). Sustentabilidade: Óleo essencial de pimentarosa de acordo com a maturação das sementes. Produtos Florestais Não Madeireiros Tecnologia, Mercado, Pesquisas e Atualidades, Editora Científica, cap. 15. DOI: $10.37885 / 210102794$

Picolloto, A.M.; Ariati, M.; Franciscato, L. M. S. S.; Bittencourt, E. B. C.; Sakai, O. A.; Moritz, C. M. F. (2020). Chemical and thermoanalytical characterization of the pink pepper (Schinus terebinthifolius Raddi) Seeds Essential Oil, Revista Mundi Engenharia, Tecnologia e Gestão. Paranaguá, PR, v.5, n.7, p. 286-01, 286-21, DOI:

$10.21575 / 25254782$ rmetg2020vol5n71120

Queiroz, S. C. N.; Collins C.H. e Jardim I. C. S. F., (2001). Métodos de extração e/ou concentração de compostos encontrados em fluidos biológicos para posterior determinação cromatográfica. Química Nova. v. 24, n. 1, p. 68-76. DOI: 10.1590/S010040422001000100013

Santos, M. R. G. dos; Silva, J. H. S. da; Caxito, M. L. do C., (2015). Brief review on the medicinal uses and antimicrobial activity of different parts of Schinus terebinthifolius Raddi. International Journal of Pharmacy and Pharmaceutical Sciences, v. 7, n. 12, p. 1-7. https://innovareacademics.in/journals/index.php/ijpps/article/view/8192

Shahidi, F.; Ambigaipalan, P., (2015). Phenolics and polyphenolics in foods, beverages and spices: Antioxidant activity and health effects - A review. Journal of Functional Foods, v. 18, p. 820-897. https://www.sciencedirect.com/science/article/pii/S1756464615003023

Silva, A. G.; Almeida, D. L.; Ronchi, S. N.; Bento, A. C.; Scherer, R.; Ramos, A. C.; Cruz, Z. M. A., (2010). The essential oil of Brazilian pepper, Shinus terebinthifolius raddi in larval control of Stegomyia aegypti (Linnaeus, 1762). Parasites \& Vectors. v. 3, n. 79, p. 79 - 83. DOI: $10.1186 / 1756-3305-4-129$

SILVA, Geisiane Rosa da. Estudo de formação e estabilidade de nanopartículas de poliácido lático para liberação controlada do óleo essencial de Shinus Molle L. (2015). Dissertação (Mestrado em Desenvolvimento, Caracterização e Aplicação de Materiais) - Escola de 


\section{DUISUl}

Engenharia de São Carlos, University of São Paulo, São Carlos, 2015. doi:10.11606/D.18.2015.tde-03092015-141731. Acesso em: 2021-09-08.

Silva-Neto, R. S.; Araújo, R. S.; Pessoa, C. V., (2018). Shinus terebinthifolius raddi: propriedades antimicrobiana, antiinflamatória e cicatrizante. Mostra Científica da Farmácia. v. 4, n. 2. http://reservas.fcrs.edu.br/index.php/mostracientificafarmacia/article/view/2352

Simões, L. M. S., 2018. Desenvolvimento de estratégias para a valorização de uma planta endógena da costa marítima portuguesa: a Corema álbum (L.) D. Don. Dissertação (Mestrado em Biotecnologia), Coimbra.

https://comum.rcaap.pt/bitstream/10400.26/28200/1/Relatorio\%20de\%20Est\%c3\%a1 gio\%20 Profissionalizante $\% 20 \mathrm{em} \% 20 \mathrm{Biotecnologia} \% 2 \mathrm{c} \% 20$ Desenvolvimento $\% 20 \mathrm{de} \% 20 \mathrm{Estrat} \% \mathrm{c} 3$ \%a9gias\%20para\%20a\%20Valoriza\%c3\%a7\%c3\%a3o\%20de\%20uma\%20Planta 1.pdf

Uliana, M. P.; Fronza, M.; Silva, A. G. da; Vargas, T. S.; Andrade, T. U. de; Scherer, R., 2016. Composition and biological activity of Brazilian rose pepper (Schinusterebinthifolius Raddi) leaves. Industrial Crops and Products. v. 83, p. 235-240. https://www.sciencedirect.com/science/article/abs/pii/S0926669015305756 